\title{
A UNIFIED APPROACH TO COMPUTE FOLIATIONS, INERTIAL MANIFOLDS, AND TRACKING SOLUTIONS
}

\author{
Y.-M. CHUNG AND M. S. JOLLY
}

\begin{abstract}
Several algorithms are presented for the accurate computation of the leaves in the foliation of an ODE near a hyperbolic fixed point. They are variations of a contraction mapping method used by Ricardo Rosa in 1995 to compute inertial manifolds, which represents a particular leaf in the unstable foliation. Such a mapping is combined with one for the leaf in the stable foliation to compute tracking solutions. The algorithms are demonstrated on the Kuramoto-Sivashinsky equation.
\end{abstract}

\section{INTRODUCTION}

The Hartman-Grobman Theorem provides a local foliation for an ODE near a hyperbolic point; through each nearby (base) point there is a pair of leaves that define a conjugacy to the linearized flow. In the classic case where the base point is the hyperbolic point itself, one leaf is its unstable manifold, the other its stable manifold. In that case the leaves are invariant; for a general base point they are not. They can, however, be characterized by the exponential growth/decay rates of the differences between solutions that start on them. If the gap in the spectrum of the linear part sufficiently dominates the Lipschitz constant for the nonlinear part in a large enough neighborhood, and the spectrum is positioned properly, the unstable manifold is an inertial manifold. Each solution is attracted at an exponential rate to a particular "tracking" solution on the inertial manifold. We present several algorithms for the accurate computation of the leaves in the foliation and also for the tracking initial condition for a given solution. The algorithms are demonstrated on the Kuramoto-Sivashinsky equation, which is an amplitude model of thin film flow (see 14] and references therein).

There has been considerable analysis of foliations in the literature. The finite dimensional case was studied in 18, followed by treatments for particular partial differential equations (PDEs) in 20] and 1. The exponential tracking property of inertial manifolds was established in 12 . We consider here the general Banach space setting, as in [2], [3, and [6], and follow the particular framework in [5].

Received by the editor September 25, 2012 and, in revised form, September 12, 2013 and October 27, 2013.

2010 Mathematics Subject Classification. Primary 34C40, 34C45, 37L25.

Key words and phrases. Foliations, inertial manifolds, tracking solution, spectral gap condition.

This work was supported in part by NSF grant numbers DMS-1008661 and DMS-1109638. The authors thank Ricardo Rosa for several stimulating discussions, and the referees for their helpful comments. 
The computation of different elements in a foliation have been treated separately with a variety of approaches. A series of papers culminating in 4. presents a parametrization approach to invariant manifolds. The survey paper [19] discusses a great number of methods for classic stable and unstable 2D manifolds. Those manifolds are global, but are generally not the graphs of functions, unlike inertial manifolds, which are usually assumed to be both [11. Approximate inertial manifolds (see e.g. 14]) for dissipative PDEs are explicit expressions for the enslavement of the high modes in terms of the low modes. To reduce the error in their approximation one must increase the number of low modes, and hence the dimension of the manifold. Direct computation of a global inertial manifold of fixed dimension was carried out in [25, while accurate evaluation of the enslavement at individual low mode inputs was achieved in [16,22]. The first efforts to compute tracking solutions appear in [8, 23, 24, in the context of center manifolds. Those methods involve an expansion of normal forms and iterative procedure to project onto a local basis of the tangent space of the center manifold.

We present here a unified approach to computing all these elements. It is based on the Lyapunov-Perron contraction mapping on spaces of functions in time used in [5], and outlined in Section 1. The fixed point of the mapping is a particular solution of the differential equation whose initial value provides an enslavement of either the high modes in terms of the low, in the case of a leaf in the unstable foliation, or vice versa in the the case of the stable foliation. These leaves are manifolds and graphs of functions whose Lipschitz constants are less than one if the linear term in the equation sufficiently dominates the nonlinear term. Both functions are then combined to form yet another contraction mapping whose fixed point is the intersection of the manifolds. In the particular case where the leaf in the unstable foliation passes through a steady state (as in the inertial manifold), the fixed point of the combined contraction mapping is the tracking solution for any point on the leaf in the stable foliation.

The key then, is to discretize the Lyapunov-Perron contraction mapping. This was first done in 26] for the particular case of the inertial manifold by using piecewise constant functions over increasingly finer time intervals. We adapt that approach to the stable foliation which requires an inner integration of the differential equation, which happens to be forward in time, so it is practical for PDEs. This is done in Section 2. We then consider improvements based on Aitken's acceleration and Simpson's method. The new methods are applied to the particular case of an inertial manifold in Section 3, and then combined for stable and unstable foliations to compute tracking solutions in Section 4. We wrap up in Section 5 with a comparison of long time dynamics of computed tracking solutions and linearly projected initial conditions. Public domain software for this approach is available at [7. While this paper concerns autonomous differential equations, the LyapunovPerron method is also applicable in the nonautonomous case, which we will treat in future work.

\section{Assumptions And foliation theory}

We recall here the main features of foliation theory for ODEs, following the presentation by Castaneda and Rosa [5], where the proof can be found. Let $X$ and $Y$ be Banach spaces and $Z=X \times Y$ be endowed with the norm $\|z\|=\|(x, y)\|=$ $\max \{\|x\|,\|y\|\}$. Let $F: Z \longrightarrow X$ and $G: Z \longrightarrow Y$ and $A, B$ be two linear bounded 
operators defined on $X$ and $Y$. Consider the following system of ODEs:

$$
\begin{aligned}
& \dot{x}=A x+F(x, y), \\
& \dot{y}=B y+G(x, y) .
\end{aligned}
$$

The assumptions are the following:

$$
\begin{aligned}
& \left\|e^{A t}\right\| \leq e^{\alpha t} \text { and }\left\|e^{-B t}\right\| \leq e^{-\beta t}, \forall t \geq 0, \text { and for some } \alpha, \beta \in \mathbb{R} . \\
& H(z)=(F(z), G(z)) \text { is Lipschitz with } \operatorname{Lip}(H) \leq \delta \text { and } H(0)=0 . \\
& 2 \delta<\beta-\alpha, \text { called the spectral gap condition. }
\end{aligned}
$$

Since $H(z)$ is Lipschitz and $A, B$ are bounded operators, it is known that the autonomous differential equation

$$
\dot{z}=C z+H(z),
$$

where $C=A \times B$ possesses a global unique solution for any given initial condition. We denote by $z\left(t, z_{0}\right)$, the solution of (1.5) with initial condition $z(0)=z_{0} \in Z$, and $x\left(t, z_{0}\right)$ and $y\left(t, z_{0}\right)$, the $X$ and $Y$ components of $z\left(t, z_{0}\right)$, respectively. Thus, $z\left(t, z_{0}\right)=\left(x\left(t, z_{0}\right), y\left(t, z_{0}\right)\right)$, for all $t \in \mathbb{R}$ and $z_{0} \in Z$.

Typically, the nonlinear terms in most physical models are not globally Lipschitz. If this is the case and the system is dissipative, the nonlinear terms can be truncated outside the absorbing ball. More precisely, let $\rho$ be the radius of the absorbing ball. Consider the prepared equation

$$
\dot{z}=C z+H_{\rho}(z),
$$

where $H_{\rho}: Z \rightarrow Z$ which agrees with $H$ for $\|z\| \leq \rho$ and is globally Lipschitz. Since all the long time behavior of the original system is in the absorbing ball, such a preparation leaves that behavior unchanged. One choice of $H_{\rho}$ is

$$
H_{\rho}=\theta_{\rho}(\|z\|) H(z),
$$

with

$$
\theta_{\rho}(r)=\theta\left(\frac{r^{2}}{\rho^{2}}\right), \quad \theta(s)=\left\{\begin{array}{l}
1, \text { for } s \in[0,1] \\
2(s-1)^{3}-3(s-1)^{2}+1, \text { for } s \in[1,2] \\
0, \text { for } s>2
\end{array}\right.
$$

The main result in [5] is to characterize foliations by the exponential growth/ decay of the difference of any two solutions with initial data in the same leaf.

Theorem 1.1 (Foliation Theorem). (1) (Stable) $Z=\bigcup_{y \in Y} \mathcal{M}_{y}$, where

(i) $\mathcal{M}_{y}=\left\{z_{0} \in Z:\left\|z\left(t, z_{0}\right)-z(t,(0, y))\right\| \leq\left\|z_{0}-(0, y)\right\| e^{(\alpha+\delta) t}\right.$, $\forall t \geq 0\}$

(ii) $\mathcal{M}_{y}=\operatorname{graph}\left(\Phi_{y}\right)$, for some $\Phi_{y}: X \rightarrow Y$ such that $\Phi_{y}(0)=y$.

(2) (Unstable) $Z=\bigcup_{x \in X} \mathcal{N}_{x}$, where

(i) $\mathcal{N}_{x}=\left\{z_{0} \in Z:\left\|z\left(t, z_{0}\right)-z(t,(x, 0))\right\| \leq\left\|z_{0}-(x, 0)\right\| e^{(\beta-\delta) t}, \forall t \leq 0\right\}$

(ii) $\mathcal{N}_{x}=\operatorname{graph}\left(\Psi_{x}\right)$, for some $\Psi_{x}: Y \rightarrow X$ such that $\Psi_{x}(0)=x$.

(3) Both $\Phi_{y}$ and $\Psi_{x}$ have Lipschitz constants bounded by $\delta /(\beta-\alpha-\delta)$.

The terminology stable foliation (unstable foliation) comes from the classic case where $\alpha<0<\beta$, in which $\mathcal{M}_{0}\left(\mathcal{N}_{0}\right)$ are respectively the stable (unstable) manifolds 
of 0 . The framework, however, also applies if $\alpha<\beta<0$ or $0<\alpha<\beta$. Regardless, $\mathcal{M}_{0}$ and $\mathcal{N}_{0}$ are both invariant;

$$
z_{0} \in \mathcal{M}_{0} \Rightarrow z\left(t, z_{0}\right) \in \mathcal{M}_{0} \forall t \in \mathbb{R},
$$

and similarly for $\mathcal{N}_{0}$. As a consequence, given any initial data $z_{0} \in Z$, properties (i) in Theorem 1.1 define distinguished solutions in $\mathcal{M}_{0}$ and $\mathcal{N}_{0}$.

Proposition 1.2 (Exponential Tracking). Given $z_{0} \in Z$, there exists a unique $z_{0}^{+} \in \mathcal{N}_{0}$,

$$
\left\|z\left(t, z_{0}\right)-z\left(t, z_{0}^{+}\right)\right\| \leq e^{(\alpha+\delta) t}\left\|z_{0}-z_{0}^{+}\right\|, \quad \forall t \geq 0 .
$$

Definition 1.3. The solution $z\left(\cdot, z_{0}^{+}\right)$is called the exponential tracking of $z\left(\cdot, z_{0}\right)$ and $z_{0}^{+}$is called the tracking solution of $z_{0}$.

By Theorem 1.1, the entire foliation is established. The intersection of a leaf from the stable foliation with one from the unstable foliation is a single element in $Z$; more precisely, for each $x_{1} \in X, y_{1} \in Y, \mathcal{M}_{y_{1}} \cap \mathcal{N}_{x_{1}}$ is a single element of $Z$. This is proved in [5] by showing that

$$
\Sigma: Z \rightarrow Z, \Sigma:(x, y) \mapsto\left(\Psi_{x_{1}}(y), \Phi_{y_{1}}(x)\right),
$$

has a unique fixed point, which is the intersection of two manifolds. In Section 4 , we will implement an approximate of the $\Sigma$ map iteratively to compute the tracking solution.

If

$$
\alpha+\delta<0 \text { and } \operatorname{dim}(Y)<\infty
$$

then $\mathcal{N}_{0}$ is an inertial manifold, i.e., an exponentially attracting, finite dimensional, Lipschitz manifold. Some of the key features of the foliation are illustrated in Figure 1(A).

1.1. An example: test problem. In this section, we will give an example that will be used to demonstrate the algorithms we developed in this article. Consider the simplest system:

$$
\frac{d}{d t}\left(\begin{array}{l}
\tilde{x} \\
\tilde{y}
\end{array}\right)=\left(\begin{array}{c}
-\tilde{x} \\
\tilde{y}
\end{array}\right) .
$$

The foliation of the linear system (1.10) consists of vertical and horizontal lines. In order to obtain a nontrivial foliation, we will apply the transformation $T=T_{2} \circ T_{1}$, where

$$
T_{1}\left(\begin{array}{c}
\tilde{x} \\
\tilde{y}
\end{array}\right)=\left(\begin{array}{c}
\tilde{x}+\frac{\tilde{y}}{p \sqrt{1+\tilde{y}^{2}}} \\
\tilde{y}
\end{array}\right), \quad T_{2}\left(\begin{array}{c}
\tilde{x} \\
\tilde{y}
\end{array}\right)=\left(\begin{array}{c}
\tilde{x} \\
\tilde{y}+\frac{1}{p} \tan ^{-1}(\tilde{x})
\end{array}\right) .
$$

By an elementary calculation we obtain

$$
\left(\begin{array}{l}
x \\
y
\end{array}\right)=T\left(\begin{array}{l}
\tilde{x} \\
\tilde{y}
\end{array}\right)=\left(\begin{array}{c}
\tilde{x}+\frac{\tilde{y}}{p \sqrt{1+\tilde{y}^{2}}} \\
\tilde{y}+\frac{1}{p} \tan ^{-1}\left(\tilde{x}+\frac{\tilde{y}}{p \sqrt{1+\tilde{y}^{2}}}\right)
\end{array}\right) .
$$




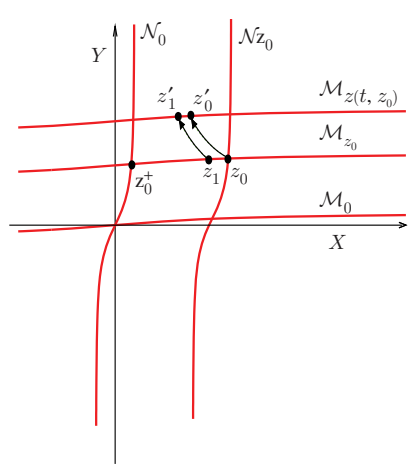

(A) Foliations for (1.13). $z_{i}^{\prime}:=z\left(t_{0}, z_{i}\right)$ for $i=0$, and 1.

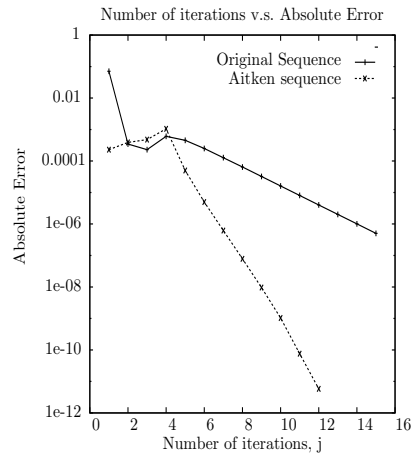

(B) Number of iterations versus the absolute error.

\begin{tabular}{|l|l|l|}
\hline$j$ & SIMP & SIMPGS \\
\hline 1 & $0.2699 \mathrm{E}-1$ & $0.9353 \mathrm{E}-7$ \\
\hline 2 & $0.3128 \mathrm{E}-5$ & $0.4832 \mathrm{E}-6$ \\
\hline 3 & $0.2435 \mathrm{E}-5$ & $0.4831 \mathrm{E}-6$ \\
\hline 4 & $0.4836 \mathrm{E}-6$ & $0.4831 \mathrm{E}-6$ \\
\hline 5 & $0.4830 \mathrm{E}-6$ & $0.4831 \mathrm{E}-6$ \\
\hline 6 & $0.4831 \mathrm{E}-6$ & $0.4831 \mathrm{E}-6$ \\
\hline 7 & $0.4831 \mathrm{E}-6$ & $0.4831 \mathrm{E}-6$ \\
\hline
\end{tabular}

(c) Number of iterations versus the absolute error.

Figure 1. (A) $\mathcal{N}_{0}$ is the inertial manifold and $z_{0}^{+}$is the tracking solution for $z_{0} . \mathcal{M}_{z_{0}}$ is the leaf in the stable foliation through $z_{0}$ and has property that for any two points $z_{0}, z_{1}$ on $\mathcal{M}_{z_{0}}$ and any time $t>0, z\left(t, z_{0}\right)$ and $z\left(t, z_{1}\right)$ lie on the same manifold $\mathcal{M}_{z\left(t, z_{0}\right)}$. (B) Performances of PWCONST and PWCONST with Aitken's acceleration for (1.13) with parameters $p=10, \tilde{z}_{0}=(1,1)$ and $\tilde{x}=3$. (C) Performances of SIMP and SIMPGS for the same settings as (B).

After the transformation $T$, the new ODE can be written as:

$$
\begin{aligned}
\frac{d x}{d t}= & -x+\frac{y-\frac{1}{p} \tan ^{-1}(x)}{p\left(1+\left(y-\frac{1}{p} \tan ^{-1}(x)\right)^{2}\right)^{1 / 2}}+\frac{y-\frac{1}{p} \tan ^{-1}(x)}{p\left(1+\left(y-\frac{1}{p} \tan ^{-1}(x)\right)^{2}\right)^{3 / 2}} \\
\frac{d y}{d t}= & y-\frac{1}{p} \tan ^{-1}(x)+\frac{1}{p\left(1+x^{2}\right)}\left[-x+\frac{y-\frac{1}{p} \tan ^{-1}(x)}{p\left(1+\left(y-\frac{1}{p} \tan ^{-1}(x)\right)^{2}\right)^{1 / 2}}\right. \\
& \left.+\frac{y-\frac{1}{p} \tan ^{-1}(x)}{p\left(1+\left(y-\frac{1}{p} \tan ^{-1}(x)\right)^{2}\right)^{3 / 2}}\right] .
\end{aligned}
$$

Note that $p$ is a parameter that controls the Lipschitz constant. In fact, we can characterize the complete foliation. However, for the purpose of this article, we are interested in only the inertial manifold (invariant unstable manifold) and a leaf in the stable foliation. 
First consider the inertial manifold for the new system. Since the invariant unstable manifold for the original system is the $y$-axis, its image under the map $T$ is the invariant unstable manifold for the new system. That is,

$$
\left(\begin{array}{l}
x \\
y
\end{array}\right)=T\left(\begin{array}{l}
0 \\
\tilde{y}
\end{array}\right)=\left(\begin{array}{c}
\frac{\tilde{y}}{p \sqrt{1+\tilde{y}^{2}}} \\
\tilde{y}+\frac{1}{p} \tan ^{-1}\left(\frac{\tilde{y}}{p \sqrt{1+\tilde{y}^{2}}}\right)
\end{array}\right) .
$$

Since the leaf in the stable foliation through $\tilde{z_{0}}$ for the original system is the horizontal line, $\tilde{y}=\tilde{y}_{0}$ for a given $\tilde{y}_{0}$, the leaf in the stable foliation for the new system is

$$
\left(\begin{array}{l}
x \\
y
\end{array}\right)=T\left(\begin{array}{c}
\tilde{x} \\
\tilde{y}_{0}
\end{array}\right)=\left(\begin{array}{c}
\tilde{x}+\frac{\tilde{y}_{0}}{p \sqrt{1+\tilde{y}_{0}^{2}}} \\
\tilde{y}_{0}+\frac{1}{p} \tan ^{-1}\left(\tilde{x}+\frac{\tilde{y}_{0}}{p \sqrt{1+\tilde{y}_{0}^{2}}}\right)
\end{array}\right) .
$$

By substitution, one obtains

$$
y=\tilde{y}_{0}+\frac{1}{p} \tan ^{-1}(x) .
$$

Given any initial condition $\tilde{z_{0}}=\left(\tilde{x_{0}}, \tilde{y_{0}}\right)$, the tracking solution for the original system is $\left(0, \tilde{y_{0}}\right)$. Thus, the tracking solution for the new system is

$$
T\left(\begin{array}{c}
0 \\
\tilde{y_{0}}
\end{array}\right)=\left(\begin{array}{l}
x \\
y
\end{array}\right)=\left(\begin{array}{c}
\frac{1}{p \sqrt{1+\tilde{y}_{0}^{2}}} \\
\tilde{y_{0}}+\frac{1}{p} \tan ^{-1}\left(\frac{1}{p \sqrt{1+\tilde{y}_{0}^{2}}}\right)
\end{array}\right) .
$$

\section{Computation of the stable Foliation}

There are four different algorithms presented. The first, PWCONST, modifies one in [26] using piecewise constant functions to approximate the integrals in (2.1), to the more complicated case of (2.4). The second combines PWCONST with a vector version of Aitken's acceleration process. Simpson's rule is used for the approximation of the integrals in SIMP, and combined with Gauss-Seidel iteration in SIMPGS.

2.1. PWCONST algorithm. In [26, an algorithm is developed for the accurate computation of inertial manifolds under the additional assumption (1.9). The main idea is to find the fixed point of the contraction mapping

$$
\mathcal{U}(\psi, y)(t)=e^{t B} y+\int_{-\infty}^{t} e^{(t-s) A} F(\psi(s)) d s-\int_{t}^{0} e^{(t-s) B} G(\psi(s)) d s, \forall t \leq 0
$$

on the Banach space

$$
\mathcal{G}_{\sigma}=\left\{\psi \in C((-\infty, 0], Z) ;\|\psi\|_{\sigma}=\sup _{t \leq 0} e^{\sigma t}\|\psi(t)\|<\infty\right\}, \sigma \in(\alpha+\delta, \beta-\delta) .
$$

The inertial manifold is the graph of the function:

$$
\Psi(y)=P \psi(0),
$$

where $\psi$ is the fixed point of $\mathcal{U}$ and $P$ is a projector from $Z$ onto $X$. In the $j$ th iteration, $\psi$ is approximated by $\psi^{(j)}$, a function that is piecewise constant on $N_{j}$ time intervals of length $h_{j}$. It is shown in [26] that $\psi^{(j)} \rightarrow \psi$ as $j \rightarrow \infty$ provided $N_{j} h_{j} \rightarrow \infty$ (e.g. $h_{j}=2^{-j}$ and $\left.N_{j}=j 2^{j}\right)$. In this approach the integrals in $\mathcal{U}$ can be evaluated explicitly. 
To compute the leaf in the stable foliation through $z_{0}$, we follow the existence proof in [5] and approximate the fixed point of the mapping

$$
\begin{aligned}
\mathcal{T}_{z_{0}}(\varphi, x)(t)=e^{t A} x & +\int_{0}^{t} e^{(t-s) A}\left[F\left(\varphi(s)+z\left(s, z_{0}\right)\right)-F\left(z\left(s, z_{0}\right)\right)\right] d s \\
& -\int_{t}^{\infty} e^{(t-s) B}\left[G\left(\varphi(s)+z\left(s, z_{0}\right)\right)-G\left(z\left(s, z_{0}\right)\right)\right] d s
\end{aligned}
$$

on

$$
\mathcal{F}_{\sigma}=\left\{\varphi \in C([0, \infty], Z) ;\|\varphi\|_{\sigma}=\sup _{t \geq 0} e^{-\sigma t}\|\varphi(t)\|<\infty\right\}, \sigma \in(\alpha+\delta, \beta-\delta) .
$$

Let $\varphi$ be the fixed point of $\mathcal{T}_{z_{0}}$. The leaf in the stable foliation through $z_{0}$ is the graph of the function

$$
\Phi_{z_{0}}(x)=y_{0}+Q \varphi\left(x-x_{0}\right)(0),
$$

where $Q=I-P$. We modify the algorithm in [26] to fit $\mathcal{T}$. The two main differences are: 1. one needs to solve for the ODE forward in time - we use a fourth order Runge-Kutta method (RK4); 2. an additional function evaluation is needed. The rate of convergence is linear so that we can use the Aitken acceleration process, as discussed in the next section, to gain a better approximation. Since piecewise constant functions are used in this algorithm, we denote it by PWCONST.

2.1.1. Aitken acceleration. Aitken's acceleration, also known as Aitken's $\Delta^{2}$ process, is used for accelerating the rate of convergence of a sequence. The method works if one has a linear rate of convergence sequence.

Definition 2.1. Given a sequence $\left\{x_{k}\right\}_{k=0}^{\infty}$ in $\mathbb{R}$, Aitken's acceleration sequence, $\left\{\mathcal{A} x_{k}\right\}_{k=0}^{\infty}$, is defined as

$$
\mathcal{A} x_{k}=x_{k}-\frac{\left(\Delta x_{k}\right)^{2}}{\Delta^{2} x_{k}}
$$

where $\Delta x_{k}:=x_{k+1}-x_{k}$ and $\Delta^{2} x_{k}:=\Delta x_{k+1}-\Delta x_{k}$.

This is the classic Aitken acceleration for a sequence in $\mathbb{R}$. We will also apply a vector version found in [21].

Definition 2.2. Given a sequence $\left\{z_{k}\right\}_{k=0}^{\infty}$ in $\mathbb{R}^{n}$, define

$$
\Delta z_{k}:=\left(z_{k+1}-z_{k}, \ldots, z_{k+n}-z_{k+n-1}\right) \text { and } \Delta^{2} z_{k}:=\Delta z_{k+1}-\Delta z_{k} .
$$

Aitken's acceleration sequence for $\mathbb{R}^{n}$ is defined as follows:

$$
\mathcal{A} z_{k}=z_{k}-\left(\Delta z_{k}\right)\left(\Delta^{2} z_{k}\right)^{-1}\left(z_{k+1}-z_{k}\right) .
$$

Note that both $\Delta z_{k}$ and $\Delta^{2} z_{k}$ are matrices of size $n \times n$.

The advantage of the Aitken sequence is that it converges much faster to the limit than the original sequence does. Moreover, computing the Aitken sequence is much cheaper than computing the original sequence because it is applied to elements in phase space $z_{k} \in Z$, rather than (a discretized version of) the function space on which $\mathcal{U}, \mathcal{T}$ act; it amounts to post-processing the original sequence. A disadvantage is that it requires $n+k$ iterations of the original mapping $\mathcal{U}(\mathcal{T})$ to produce $k$ terms in the Aitken sequence, which may be prohibitive if $n$, the dimension of $Z$, is large. We will see these numerical results in the next section. 
2.2. SIMP algorithm. First, we give a recursive relation for $\mathcal{T}_{z_{0}}(\varphi, x)$ in the time variable. Second, we introduce the main algorithm. At the end of this section, numerical results for the test problem (1.13) will be given.

Let $\left\{t_{i}\right\}_{0}^{N}$ be the uniform partition of a time interval and let $h:=t_{i+1}-t_{i}$. Let $P$ be the projector from $Z$ onto $X$, i.e., $P z=x$, where $z=(x, y)$ and $Q=I-P$.

2.2.1. A recursive relation. We state the recursive relations in the following proposition.

\section{Proposition 2.3.}

(i) For $i=1,2, \ldots, N-1$, the $X$-component of $\mathcal{T}_{z_{0}}(\varphi, x)$ is

$$
\begin{aligned}
P \mathcal{T}_{z_{0}}(\varphi, x)\left(t_{i+1}\right)= & e^{\left(t_{i+1}-t_{i-1}\right) A} P \mathcal{T}_{z_{0}}(\varphi, x)\left(t_{i-1}\right) \\
& +\int_{t_{i-1}}^{t_{i+1}} e^{\left(t_{i+1}-s\right) A}\left[F\left(\varphi(s)+z\left(s, z_{0}\right)\right)-F\left(z\left(s, z_{0}\right)\right)\right] d s,
\end{aligned}
$$

where $P \mathcal{T}_{z_{0}}(\varphi, x)\left(t_{0}\right)=x$ and

$$
P \mathcal{T}_{z_{0}}(\varphi, x)\left(t_{1}\right)=e^{t_{1} A} x+\int_{0}^{t_{1}} e^{\left(t_{1}-s\right) A}\left[F\left(\varphi(s)+z\left(s, z_{0}\right)\right)-F\left(z\left(s, z_{0}\right)\right)\right] d s .
$$

(ii) For $i=N-1, N-2, \ldots, 1$, the $Y$-component of $\mathcal{T}_{z_{0}}(\varphi, x)$ is

$$
\begin{aligned}
Q \mathcal{T}_{z_{0}}(\varphi, x)\left(t_{i-1}\right)= & e^{\left(t_{i-1}-t_{i+1}\right) B} Q \mathcal{T}_{z_{0}}(\varphi, x)\left(t_{i+1}\right) \\
& -\int_{t_{i-1}}^{t_{i+1}} e^{\left(t_{i-1}-s\right) B}\left[G\left(\varphi(s)+z\left(s, z_{0}\right)\right)-G\left(z\left(s, z_{0}\right)\right)\right] d s,
\end{aligned}
$$

where $Q \mathcal{T}_{z_{0}}(\varphi, x)\left(t_{N}\right)=-\int_{t_{N}}^{\infty} e^{\left(t_{N}-s\right) B}\left[G\left(\varphi(s)+z\left(s, z_{0}\right)\right)-G\left(z\left(s, z_{0}\right)\right)\right] d s$ and

$$
\begin{aligned}
& Q \mathcal{T}_{z_{0}}(\varphi, x)\left(t_{N-1}\right)=Q \mathcal{T}_{z_{0}}(\varphi, x)\left(t_{N}\right) e^{\left(t_{N-1}-t_{N}\right) B} \\
& \quad-\int_{t_{N-1}}^{t_{N}} e^{\left(t_{N-1}-s\right) B}\left[G\left(\varphi(s)+z\left(s, z_{0}\right)\right)-G\left(z\left(s, z_{0}\right)\right)\right] d s .
\end{aligned}
$$

The proof of Proposition 2.3 is similar to the derivation of the recursive algorithm in $[15$.

2.2.2. The SIMP algorithms. The integrals appearing in the recursive relation can be better approximated by Simpson's rule. In order to have a consistent order, one needs to adjust the first two approximations, i.e., $P \mathcal{T}_{z_{0}}(\varphi, x)\left(t_{0}\right), P \mathcal{T}_{z_{0}}(\varphi, x)\left(t_{1}\right)$, $Q \mathcal{T}_{z_{0}}(\varphi, x)\left(t_{N}\right), Q \mathcal{T}_{z_{0}}(\varphi, x)\left(t_{N-1}\right)$

Since $P \mathcal{T}_{z_{0}}(\varphi, x)\left(t_{0}\right)=x$, no error is introduced at $t=t_{0}$. When $t=t_{1}$,

$$
P \mathcal{T}_{z_{0}}(\varphi, x)\left(t_{1}\right)=e^{t_{1} A} x+\int_{0}^{t_{1}} e^{\left(t_{1}-s\right) A}\left[F\left(\varphi(s)+z\left(s, z_{0}\right)\right)-F\left(z\left(s, z_{0}\right)\right)\right] d s,
$$


which involves the integral we need to approximate so that the error is the same as the one introduced by Simpson's rule. One can rewrite the integral as

$$
\begin{aligned}
\int_{t_{0}}^{t_{1}} e^{\left(t_{1}-s\right) A}\left[F\left(\varphi(s)+z\left(s, z_{0}\right)\right)-F\left(z\left(s, z_{0}\right)\right)\right] d s \\
=\int_{t_{0}}^{t_{3}} e^{\left(t_{1}-s\right) A}\left[F\left(\varphi(s)+z\left(s, z_{0}\right)\right)-F\left(z\left(s, z_{0}\right)\right)\right] d s \\
\quad-\int_{t_{1}}^{t_{3}} e^{\left(t_{1}-s\right) A}\left[F\left(\varphi(s)+z\left(s, z_{0}\right)\right)-F\left(z\left(s, z_{0}\right)\right)\right] d s .
\end{aligned}
$$

For the integral (2.9), Simpson's 3/8 rule is used. It has the same order error as the classic form of Simpson's rule, which is used for the integral (2.10).

The term $Q \mathcal{T}_{z_{0}}(\varphi, x)\left(t_{N}\right)$ is the tail of the convergent improper integral. We will give an estimate on $t_{N}$ so that the truncation error will be the same as the error of Simpson's rule. To do so, we need an estimate on $\varphi$, the fixed point of $\mathcal{T}_{z_{0}}(\varphi, x)$.

Lemma 2.4. Let $\varphi$ be the fixed point of $\mathcal{T}_{z_{0}}$. Then $\|\varphi\|_{\sigma} \leq \frac{\|x\|}{1-\kappa}$, where $\kappa=$ $\max \left\{\frac{\delta}{\beta-\sigma}, \frac{\delta}{\sigma-\alpha}\right\}$.

Proof. From [5], we have

$$
\left\|\mathcal{T}_{z_{0}}(\varphi)\right\|_{\sigma} \leq\|x\|+\kappa\|\varphi\|_{\sigma}
$$

for any $\varphi \in \mathcal{F}_{\sigma}$. Since $\varphi$ is the fixed point of $\mathcal{T}_{z_{0}}$, the left hand side of (2.11) can be replaced by $\|\varphi\|_{\sigma}$. By the gap condition (1.4),$\kappa<1$. Thus, by direct calculation, one has

$$
\|\varphi\|_{\sigma} \leq \frac{\|x\|}{1-\kappa}
$$

We are ready to give an estimate for $Q \mathcal{T}_{z_{0}}(\varphi, x)\left(t_{N}\right)$. Note that

$$
\begin{aligned}
\left\|Q \mathcal{T}_{z_{0}}(\varphi, x)\left(t_{N}\right)\right\| & =\left\|\int_{t_{N}}^{\infty} e^{\left(t_{N}-s\right) B}\left[G\left(\varphi(s)+z\left(s, z_{0}\right)\right)-G\left(z\left(s, z_{0}\right)\right)\right] d s\right\| \\
& \leq \int_{t_{N}}^{\infty} e^{\left(t_{N}-s\right) \beta} \delta|\varphi(s)| d s \leq \int_{t_{N}}^{\infty} e^{\left(t_{N}-s\right) \beta} \delta e^{\sigma s}\|\varphi\|_{\sigma} d s \\
& =\delta\|\varphi\|_{\sigma} e^{t_{N} \beta} \int_{t_{N}}^{\infty} e^{(\sigma-\beta) s} d s=\frac{\delta}{\beta-\sigma} e^{\sigma t_{N}}\|\varphi\|_{\sigma} \\
& \leq \frac{\delta}{\beta-\sigma} e^{\sigma t_{N}} \frac{\|x\|}{1-\kappa} \leq \frac{\kappa}{1-\kappa} e^{\sigma t_{N}}\|x\| .
\end{aligned}
$$

Let $h=t_{1}-t_{0}$. Since the error for Simpson's rule is of order $\mathcal{O}\left(h^{5}\right)$, we take

$$
\frac{\kappa}{1-\kappa} e^{\sigma t_{N}}\|x\| \leq h^{5}
$$

Hence, we choose $t_{N}$ so that

$$
e^{\sigma t_{N}} \leq \frac{1-\kappa}{\kappa\|x\|} h^{5}
$$


For $Q \mathcal{T}_{z_{0}}(\varphi, x)\left(t_{N-1}\right)$, the technique is similar to the one used for $P \mathcal{T}_{z_{0}}(\varphi, x)\left(t_{1}\right)$. Rewrite $Q \mathcal{T}_{z_{0}}(\varphi, x)\left(t_{N-1}\right)$ as two integrals:

$$
\begin{aligned}
\int_{t_{N-1}}^{t_{N}} & e^{\left(t_{N-1}-s\right) B}\left[G\left(\varphi(s)+z\left(s, z_{0}\right)\right)-G\left(z\left(s, z_{0}\right)\right)\right] d s \\
= & \int_{t_{N-3}}^{t_{N}} e^{\left(t_{N-1}-s\right) B}\left[G\left(\varphi(s)+z\left(s, z_{0}\right)\right)-G\left(z\left(s, z_{0}\right)\right)\right] d s \\
& -\int_{t_{N-3}}^{t_{N-1}} e^{\left(t_{N-1}-s\right) B}\left[G\left(\varphi(s)+z\left(s, z_{0}\right)\right)-G\left(z\left(s, z_{0}\right)\right)\right] d s .
\end{aligned}
$$

As before, the integral (2.13) can be approximated by Simpson's $3 / 8$ rule; the integral (2.14) can be approximated by Simpson's rule. Hence, for $Q \mathcal{T}_{z_{0}}(\varphi, x)\left(t_{N-1}\right)$ the error is of order $\mathcal{O}\left(h^{5}\right)$.

Before we present the algorithm, we remark on successive iteration. We have to ensure that the initial guess, $\varphi^{0}$, is in the space $\mathcal{F}_{\sigma}$ defined in (2.5). In the PWCONST algorithm, $\psi^{0}$ is simply a constant function, which is in the space $\mathcal{G}_{\sigma}$ (defined in (2.2) ) if we take $\sigma<0$ (i.e., if $\alpha+\delta<0<\beta-\delta$ ). However, in the case of the stable foliation, if $\varphi^{0}$ is constant, it may not be in $\mathcal{F}_{\sigma}$. Since $\left\|\varphi^{0}\right\|_{\sigma}=\sup _{t \geq 0} e^{-\sigma t}\left\|\varphi^{0}(t)\right\|,\left\|\varphi^{0}\right\|_{\sigma}$ is not bounded if $\sigma$ is negative. Instead of the constant $\varphi^{0}$, we take

$$
\varphi^{0}(x, t)=e^{\alpha t}\left(x-x_{0}\right) .
$$

Then

$$
\left\|\varphi^{0}\right\|_{\sigma}=\sup _{t \geq 0} e^{-\sigma t}\left\|\varphi^{0}(t)\right\|=\sup _{t \geq 0} e^{(\alpha-\sigma) t}\left\|x-x_{0}\right\|<\infty
$$

since $(\alpha-\sigma)$ is negative by the gap condition (1.4), where $\sigma \in(\alpha+\delta, \beta-\delta)$.

For the notations in the SIMP algorithm,

$$
\begin{aligned}
S\left(f_{k} ; k=i, i+1, i+2\right) & :=\frac{h}{3}\left(f_{i}+4 f_{i+1}+f_{i+2}\right), \\
S 38\left(f_{k} ; k=i, i+1, i+2, i+3\right) & :=\frac{3}{8} h\left(f_{i}+3 f_{i+1}+3 f_{i+2}+f_{i+3}\right),
\end{aligned}
$$

where $h=t_{i+1}-t_{i}$. In particular, for Simpson's rule in the $i$-loop, we have

$$
\begin{aligned}
& S\left(e^{\left(t_{i+1}-t_{k}\right) A} F_{k}^{j} ; k=i-1, i, i+1\right) \\
& =\frac{h}{3}\left[e^{\left(t_{i+1}-t_{i-1}\right) A}\left(F\left(\varphi^{j}\left(t_{i-1}\right)+z\left(t_{i-1}, z_{0}\right)\right)-F\left(z\left(t_{i-1}, z_{0}\right)\right)\right)\right. \\
& +4 e^{\left(t_{i+1}-t_{i}\right) A}\left(F\left(\varphi^{j}\left(t_{i}\right)+z\left(t_{i}, z_{0}\right)\right)-F\left(z\left(t_{i}, z_{0}\right)\right)\right) \\
& \left.+e^{\left(t_{i+1}-t_{i+1}\right) A}\left(F\left(\varphi^{j}\left(t_{i+1}\right)+z\left(t_{i+1}, z_{0}\right)\right)-F\left(z\left(t_{i+1}, z_{0}\right)\right)\right)\right] .
\end{aligned}
$$

Since $P \mathcal{T}_{z_{0}}\left(\varphi^{j+1}, x\right)\left(t_{i-1}\right)$ and $P \mathcal{T}_{z_{0}}\left(\varphi^{j+1}, x\right)\left(t_{i}\right)$ are computed in the previous steps, we could, as in Gauss-Seidel iteration, use these to obtain a better approximation. More precisely, we do the following:

$$
\begin{aligned}
& S\left(e^{\left(t_{i+1}-t_{k}\right) A} F_{k}^{j} ; k=i-1, i, i+1\right) \\
& =\frac{h}{3}\left[e^{\left(t_{i+1}-t_{i-1}\right) A}\left(F\left(P \varphi^{j+1}\left(t_{i-1}\right)+x\left(t_{i-1}, z_{0}\right), Q \varphi^{j}\left(t_{i-1}\right)+y\left(t_{i-1}, z_{0}\right)\right)-F\left(z\left(t_{i-1}, z_{0}\right)\right)\right)\right. \\
& +4 e^{\left(t_{i+1}-t_{i}\right) A}\left(F\left(P \varphi^{j+1}\left(t_{i}\right)+x\left(t_{i}, z_{0}\right), Q \varphi^{j}\left(t_{i}\right)+y\left(t_{i}, z_{0}\right)\right)-F\left(z\left(t_{i}, z_{0}\right)\right)\right) \\
& \left.+e^{\left(t_{i+1}-t_{i+1}\right) A}\left(F\left(\varphi^{j}\left(t_{i+1}\right)+z\left(t_{i+1}, z_{0}\right)\right)-F\left(z\left(t_{i+1}, z_{0}\right)\right)\right)\right] .
\end{aligned}
$$




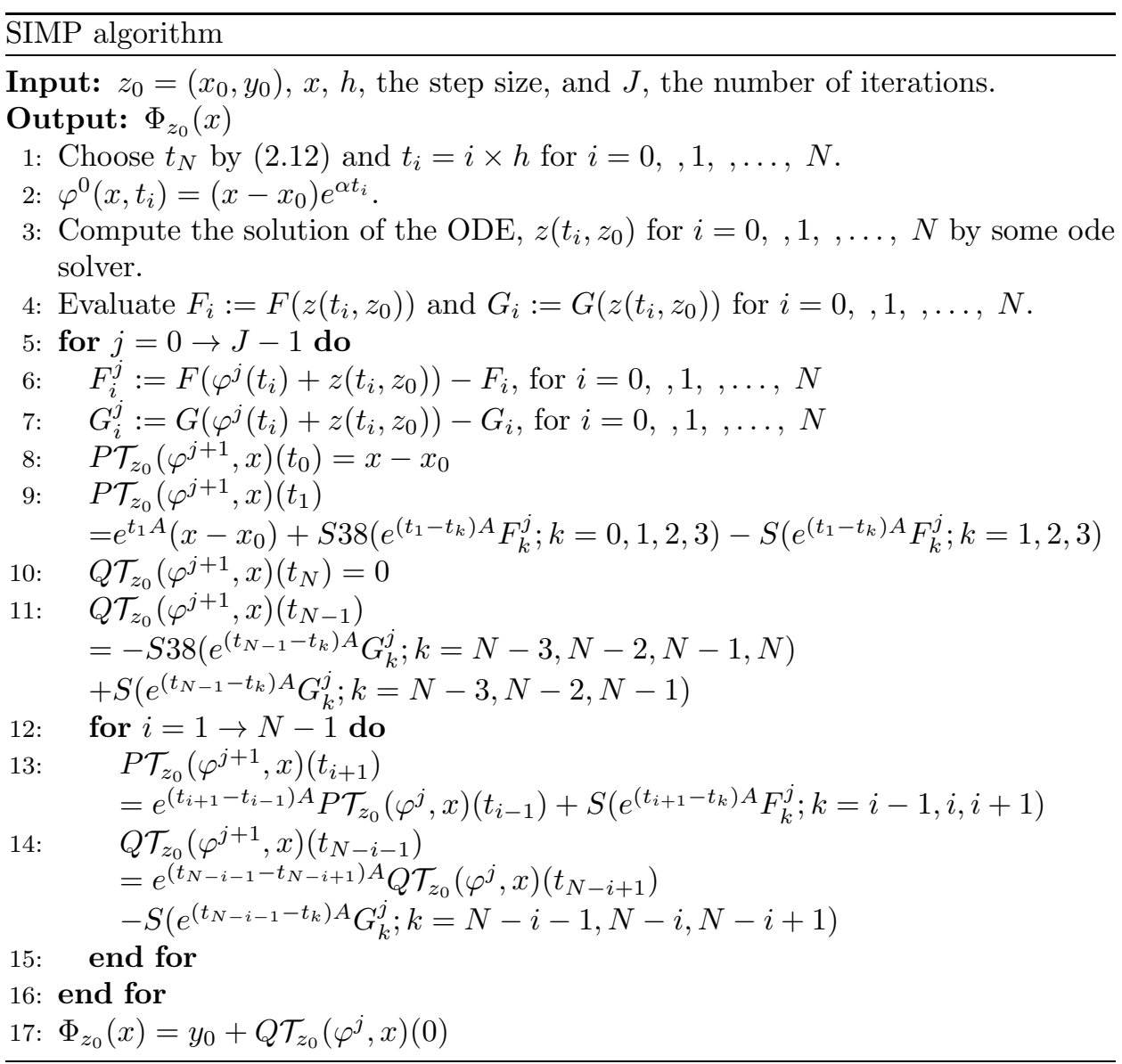

A similar partial update can be done in reverse for

$$
S\left(e^{\left(t_{N-i-1}-t_{k}\right) A} G_{k}^{j} ; k=N-i-1, N-i, N-i+1\right) .
$$

We call the resulting algorithm SIMPGS.

2.3. Numerical results for the test problem. We apply PWCONST, PWCONST+Aitken, and SIMP to compute a leaf in the stable foliation for the test problem with $p=10$. We take as the inputs for the algorithm

$$
\left(x_{0}, y_{0}\right)=T(1,1)=\left(1+\frac{1}{10 \sqrt{2}}, 1+\frac{1}{10} \tan ^{-1}\left(1+\frac{1}{10 \sqrt{2}}\right)\right)
$$

and $x=3+\frac{1}{10 \sqrt{2}}$. The output should be

$$
y=1+\frac{\tan ^{-1}(x)}{10}=1+\frac{\tan ^{-1}\left(3+\frac{1}{10 \sqrt{2}}\right)}{10} .
$$

In Figure 1(B), the error of the original sequence decreases roughly by a factor of $1 / 2$ as $j$ increases; the error of the Aitken's sequence decreases roughly by a factor of $10^{-1}$. In Figure 1(C), observe that first, both algorithms converge and both errors are of the same order. Comparing the two columns, there is a huge difference in the first two iterations. In 1 or 2 iterations, the errors from SIMPGS 
seem to be saturated while errors from SIMP are saturated in 5 or 6 iterations. This is typical. Different $h$ and different inputs give similar results.

2.4. Approximate inertial form of the KSE. The Kuramoto-Sivashinsky equation (KSE) with periodic and odd boundary conditions can be written

$$
\begin{aligned}
& \frac{\partial u}{\partial t}+4 \frac{\partial^{4} u}{\partial \xi^{4}}+\gamma\left[\frac{\partial^{2} u}{\partial \xi^{2}}+u \frac{\partial u}{\partial \xi}\right]=0 \\
& u(t, \xi)=u(t, \xi+2 \pi), \quad u(t,-\xi)=-u(t, \xi) .
\end{aligned}
$$

The solutions may be represented by the Fourier sine series

$$
u(t, \xi)=\sum_{j=-\infty}^{\infty} u_{j}(t) e^{i j \xi}=\sum_{j=1}^{\infty} b_{j}(t) \sin (j \xi),
$$

where the reality and oddness conditions on $u$ give

$$
u_{-j}=\bar{u}_{j} \text { and } u_{j}=-\frac{i b_{j}}{2} .
$$

We express the KSE in the functional form:

$$
\frac{d u}{d t}+L u+R(u)=0, \quad u \in \mathcal{H},
$$

where $\mathcal{H}$ is an appropriate Hilbert space (see [14]). The linear operator $L$ is given by

$$
L u=4 \frac{\partial^{4} u}{\partial \xi^{4}}+\gamma \frac{\partial^{2} u}{\partial \xi^{2}}
$$

along with periodic, odd boundary conditions. The remaining terms are then collected in $R$. The infinite-dimensional phase space $\mathcal{H}$ is split into low- and highwavenumber modes by means of the projectors

$$
P=P_{n}: \mathcal{H} \rightarrow \operatorname{span}\{\sin (\xi), \sin (2 \xi), \ldots, \sin (n \xi)\}, Q=Q_{n}=I-P_{n} .
$$

Thus, $u=p+q$, where $p=P u$ and $q=Q u$. We will use the approximate inertial manifold (see [9]), $\Phi_{1}(p)=-L^{-1} Q R(p)$. The approximate inertial form is

$$
\frac{d p}{d t}+L p+P R\left(p+\Phi_{1}(p)\right)=0
$$

which in our foliation framework would mean $z=p, C=-L$, and $H(z)=-P R(z+$ $\left.\Phi_{1}(z)\right)$. Compare to the Galerkin approximation which amounts to replacing $\Phi_{1}$ with $\Phi_{0} \equiv 0$. We fix $n=3$ as it was demonstrated in [14 that this is sufficient for (2.17) to capture the long time dynamics of the KSE for $\gamma \in[0,36]$. There are two reasons convergence should be slower for this reduced system. First, the gap in the eigenvalues is smaller. Second, the composition of the nonlinear term with itself in (2.17) makes for a larger Lipschitz constant.

Given two initial conditions for (2.17), denoted by $z_{1}$ and $z_{2}$, which are close to each other but on opposite sides of a separatrix, we construct leaves, $\mathcal{M}_{z_{1}}$ and $\mathcal{M}_{z_{2}}$, in the stable foliation through each point. Since each leaf is an equivalence class, the points on the same leaf should have the same long time behavior. This property is demonstrated in Figure 2. 


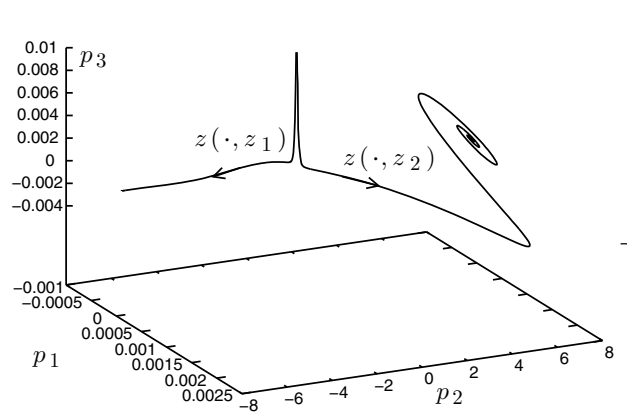

(A)

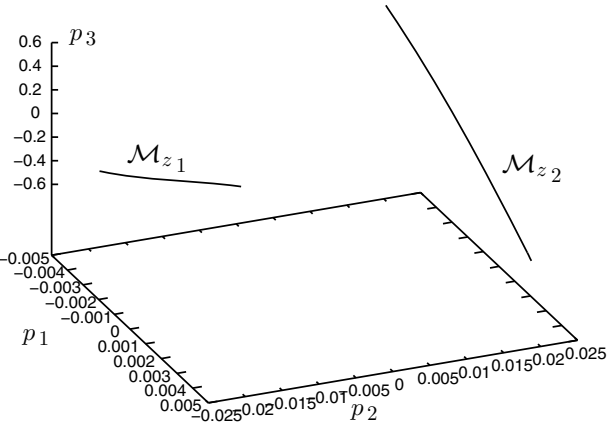

(B)

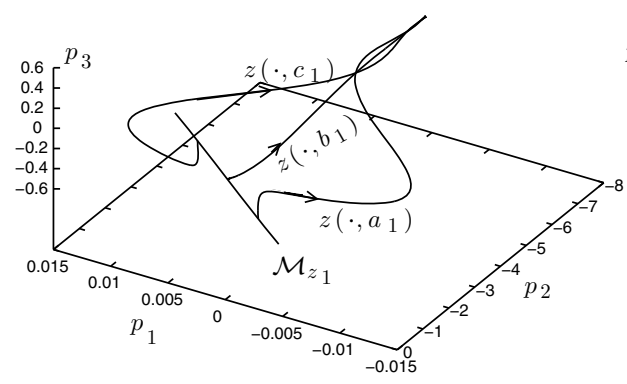

(c)

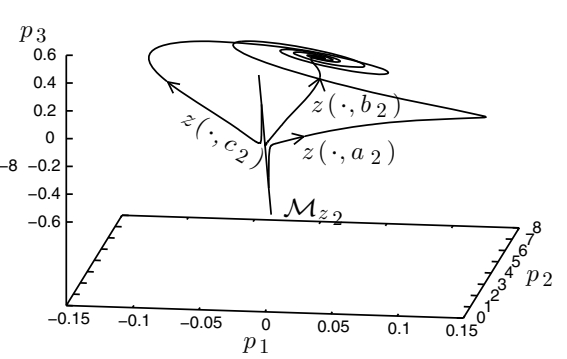

(D)

Figure 2. (A) The long time behavior of (2.17) for two nearly different initial conditions, $z_{1}=(0,0.02,0.01)$ and $z_{2}=$ $(0,-0.02,0.01)$. (B) Leaves in the stable foliation through $z_{1}$ and $z_{2}$. Note the scale of the $p_{2}$-axis. (C) $a_{1}, b_{1}, c_{1} \in \mathcal{M}_{z_{1}}$. (D) $a_{2}, b_{2}$, $c_{2} \in \mathcal{M}_{z_{2}}$.

\section{IMPROVED COMPUTATION OF INERTIAL MANIFOLDS}

Both algorithms discussed in the previous section can be adapted to compute an inertial manifold. In this section, we will show by the numerical evidence that these are the improved methods and we will apply those methods to KSE.

For the test in this section, we use a 16 mode Galerkin approximation and take $\operatorname{dim}(X)=\operatorname{dim}(Y)=8$ and $\gamma=32$. It has been shown that KSE (2.15) has an inertial manifold (see [10]) and its lowest dimension has been studied in [10, 27], and [17. In particular, at $\gamma=32$, it is shown in [17 that the computed global attractor is contained in a ball of radius 15 (in the $L^{2}$-norm) which when used in the preparation in (1.7) yields an inertial manifold of dimension five. Since a limit cycle is contained in the global attractor, which in turn is on the inertial manifold, we pick a test point, $u_{0}=x_{0}+y_{0}$, on a limit cycle and pick the low mode component, $y_{0}$, as an input of the algorithm and test how well we recover the high modes, $x_{0}$.

3.1. PWCONST plus Aitken's acceleration. As we mentioned before, since the rate of convergence is linear in PWCONST, we can apply Aitken's $\Delta^{2}$ process to accelerate the convergence. The reason there are only 5 iterations in the Aitken's sequence in Figure 3(A) is that in calculating one iteration in the Aitken's sequence, 


\begin{tabular}{|l|l|l|}
\hline$j$ & $x_{0}^{j}$ & $\mathcal{A} x_{0}^{j}$ \\
\hline 1 & $0.616 \mathrm{E}-4$ & N/A \\
\hline 2 & $0.321 \mathrm{E}-4$ & N/A \\
\hline 3 & $0.146 \mathrm{E}-4$ & N/A \\
\hline 4 & $0.707 \mathrm{E}-5$ & N/A \\
\hline 5 & $0.348 \mathrm{E}-5$ & N/A \\
\hline 6 & $0.173 \mathrm{E}-5$ & N/A \\
\hline 7 & $0.862 \mathrm{E}-6$ & N/A \\
\hline 8 & $0.430 \mathrm{E}-6$ & N/A \\
\hline 9 & $0.214 \mathrm{E}-6$ & $1.595 \mathrm{E}-11$ \\
\hline 10 & $0.107 \mathrm{E}-6$ & $1.629 \mathrm{E}-11$ \\
\hline 11 & $0.537 \mathrm{E}-7$ & $1.627 \mathrm{E}-11$ \\
\hline 12 & $0.268 \mathrm{E}-7$ & $1.627 \mathrm{E}-11$ \\
\hline 13 & $0.134 \mathrm{E}-7$ & $1.627 \mathrm{E}-11$ \\
\hline
\end{tabular}

(A) Performances of PWCONST and PWCONST + Aitken's acceleration.

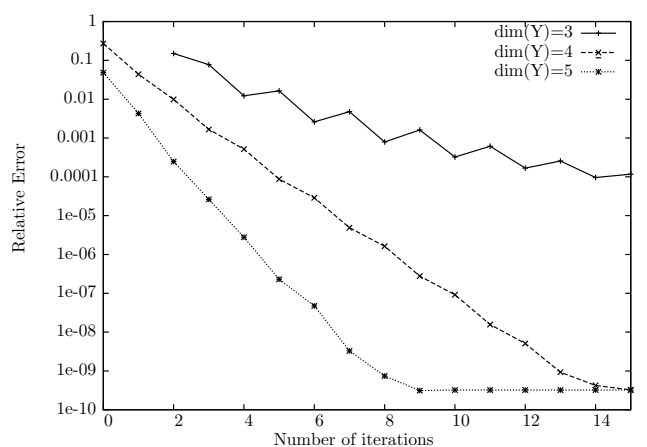

(B) Performance of the SIMP algorithm.

Figure 3. Improved computation of the inertial manifold for the 16 mode Galerkin approximation of KSE with $\gamma=32$. (A) Absolute error table for two algorithms. $x_{0}^{j}\left(\mathcal{A} x_{0}^{j}\right)$ is generated by PWCONST (PWCONST+Aitken). (B) We fix $(h, N)=(1 e-$ $6,100000)$ and vary $\operatorname{dim}(Y)$.

one needs $n+1$ iterations from the original sequence, where $n$ is the dimension of the elements of the sequence. The error of Aitken's sequence is much better than the original sequence. Another advantage of Aitken's $\Delta^{2}$ process is the cheap computation. In order to obtain a similar error with the original sequence, one needs to compute more iterations, which is more expensive than computing the Aitken's sequence. To observe the difference between these computational efforts, we compare the number of multiplications for the two algorithms, namely PWCONST and the Aitken's sequence ignoring multiplications needed for the evolution of nonlinear terms and the integrals. For the PWCONST algorithm, the required number of multiplication is at least

$$
\Sigma_{j=1}^{J} j \times 2^{j} \times 6 \times \operatorname{dim}(Z) .
$$

In this case, $\operatorname{dim}(Z)=16$. If we compute 10 terms in the PWCONST algorithm, the minimum number of multiplications is

$$
\Sigma_{j=1}^{10} j \times 2^{j} \times 6 \times \operatorname{dim}(Z)=1769664 .
$$

On the other hand, to compute one term of the Aitken's sequence, one needs to compute $\operatorname{dim}(X)$ terms in the PWCONST algorithm, solve a linear system, and calculate a matrix-vector multiplication. Thus, the number of multiplications for computing two terms of the Aitken's sequence is

$$
\sum_{j=1}^{\operatorname{dim}(X)+1} j \times 2^{j} \times 6 \times \operatorname{dim}(Z)+\left(\operatorname{dim}(X)^{3}+\operatorname{dim}(X)^{2}\right) \times 2=787776 .
$$


3.2. The SIMP algorithm. To compute the inertial manifold and a leaf through $z_{0}$ in the stable foliation is to compute the fixed point of $\mathcal{U}$ in (2.1) and $\mathcal{T}_{z_{0}}$ in (2.4) on the Banach space $\mathcal{G}_{\sigma}$ in (2.2) and $\mathcal{F}_{\sigma}$ in (2.5) respectively. These two maps are similar and in fact, if we formally replace $\left(z_{0}, \varphi, t, x, A, B, F, G\right)$ in the $\mathcal{T}$ map by $(0, \psi,-t, y, B, A, G, F)$ and drop the $z\left(s, z_{0}\right)$ terms, we obtain the $\mathcal{U}$ map. With this observation, we can easily modify the previous algorithms to compute the fixed point of the $\mathcal{U}$ map.

There are two parameters in SIMP, namely step size, $h$, and the number of points, $N$. Given $h$, one can choose $N$ by (2.12), though this requires an estimate on the Lipschitz constant of the nonlinear term; this can be found in [17. Here, we will choose $h$ and $N$ experimentally.

Next, we investigate how the spectral gap affects the algorithm. For the KSE, since the linear term is a diagonal matrix, the gap is the difference between two consecutive eigenvalues.

When $\operatorname{dim}(Y)=1$ and $\operatorname{dim}(Y)=2$, the sequences are convergent but they are not convergent to the inertial manifold. When $\operatorname{dim}(Y)=3$, the convergence is slow; it took about 50 iterations to reach the saturated error, which is about $10^{-8}$. The reason could be that the gap condition is barely satisfied for the region of phase space visited by the algorithm. As we increase $\operatorname{dim}(Y)$ and hence the gap, the convergence is faster as Figure 3(B) shows.

\section{Computation of tracking solutions}

4.1. Algorithm for tracking solution. It is shown in [5] that the exact tracking solution of a base point $z_{0}$ is the fixed point for the mapping

$$
\Sigma:(x, y) \mapsto\left(\Psi_{0}(y), \Phi_{z_{0}}(x)\right) .
$$

In Section 2 and 3, we discussed how to compute $\Psi_{0}$ and $\Phi_{z_{0}}$. Let $\Psi_{0}^{j}$ and $\Phi_{z_{0}}^{j}$ be the $j$ th approximation of $\Psi_{0}$ and $\Phi_{z_{0}}$ respectively. More precisely, let $\varphi$ and $\psi$ be the fixed points of $\mathcal{T}_{z_{0}}$ and $\mathcal{U}$ and define

$$
\Phi_{z_{0}}^{j}(x):=y_{0}+Q \varphi^{j}\left(x-x_{0}\right)(0), \quad \Psi_{0}^{j}(y):=P \psi^{j}(y)(0),
$$

where $\varphi^{j}=\mathcal{T}_{z_{0}}\left(\varphi^{j-1}\right)$ and $\psi^{j}=\mathcal{U}_{0}\left(\psi^{j-1}\right)$ for $j \geq 1$. For fixed $j_{1}$ and $j_{2}$, we then define the approximation of $\Sigma$ map by

$$
\Sigma^{j_{1}, j_{2}}:(x, y) \mapsto\left(\Psi_{0}^{j_{1}}(y), \Phi_{z_{0}}^{j_{2}}(x)\right) .
$$

As is the case for $\Sigma, \Sigma^{j_{1}, j_{2}}$ is a contraction mapping and the fixed point, $\left(x^{*}, y^{*}\right)$, is the intersection of the two manifolds that are the graphs of $\Psi_{0}^{j_{1}}$ and $\Phi_{z_{0}}^{j_{2}}$.

4.2. Convergence of the algorithm. The following results show that the algorithm for the tracking solution converges under a stronger gap condition.

Lemma 4.1. Let $\varphi^{0}(x)(t)=e^{\alpha t}\left(x-x_{0}\right), \forall t \geq 0$ and define $\varphi^{j}(x)(t)$ recursively by

$\varphi^{j}(x)(t)=\mathcal{T}_{z_{0}}\left(\varphi^{j-1}, x\right)(t)$. Then

$$
\left\|\varphi^{j}\left(x_{1}\right)-\varphi^{j}\left(x_{2}\right)\right\|_{\sigma} \leq \frac{1}{1-\kappa}\left\|x_{1}-x_{2}\right\|, \forall x_{1}, x_{2} \in X
$$

where $\kappa=\max \left\{\frac{\delta}{\beta-\sigma}, \frac{\delta}{\sigma-\alpha}\right\}$. 


\section{Proof. Consider}

$$
\begin{aligned}
& \left\|\varphi^{j}\left(x_{1}\right)(t)-\varphi^{j}\left(x_{2}\right)(t)\right\|=\left\|\mathcal{T}_{z_{0}}\left(\varphi^{j-1}, x_{1}\right)(t)-\mathcal{T}_{z_{0}}\left(\varphi^{j-1}, x_{2}\right)(t)\right\| \\
= & \| e^{t A}\left(x_{1}-x_{2}\right)+\int_{0}^{t} e^{(t-s) A}\left[F\left(\varphi^{j-1}\left(x_{1}\right)(s)+z\left(s, z_{0}\right)\right)-F\left(\varphi^{j-1}\left(x_{2}\right)(s)+z\left(s, z_{0}\right)\right)\right] d s \\
& -\int_{t}^{\infty} e^{(t-s) B}\left[G\left(\varphi^{j-1}\left(x_{1}\right)(s)+z\left(s, z_{0}\right)\right)-G\left(\varphi^{j-1}\left(x_{2}\right)(s)+z\left(s, z_{0}\right)\right)\right] d s \| \\
\leq & \max \left\{e^{\alpha t}\left\|x_{1}-x_{2}\right\|+\int_{0}^{t} e^{(t-s) \alpha} \delta\left\|\varphi^{j-1}\left(x_{1}\right)(s)-\varphi^{j-1}\left(x_{2}\right)(s)\right\| d s,\right. \\
& \left.\int_{t}^{\infty} e^{(t-s) \beta} \delta\left\|\varphi^{j-1}\left(x_{1}\right)(s)-\varphi^{j-1}\left(x_{2}\right)(s)\right\| d s\right\} .
\end{aligned}
$$

Let $\sigma \in(\alpha+\delta, \beta-\delta)$ and multiply the last inequality above by $e^{-\sigma t}$ to obtain:

$$
\begin{aligned}
& e^{-\sigma t}\left\|\varphi^{j}\left(x_{1}\right)(t)-\varphi^{j}\left(x_{2}\right)(t)\right\| \\
& \leq \max \left\{e^{(\alpha-\sigma) t}\left\|x_{1}-x_{2}\right\|+e^{-\sigma t} \int_{0}^{t} e^{(t-s) \alpha} \delta\left\|\varphi^{j-1}\left(x_{1}\right)(s)-\varphi^{j-1}\left(x_{2}\right)(s)\right\| d s,\right. \\
& \left.\quad e^{-\sigma t} \int_{t}^{\infty} e^{(t-s) \beta} \delta\left\|\varphi^{j-1}\left(x_{1}\right)(s)-\varphi^{j-1}\left(x_{2}\right)(s)\right\| d s\right\} \\
& \leq \max \left\{e^{(\alpha-\sigma) t}\left\|x_{1}-x_{2}\right\|+\delta\left\|\varphi^{j-1}\left(x_{1}\right)-\varphi^{j-1}\left(x_{2}\right)\right\|_{\sigma} \int_{0}^{t} e^{(\alpha-\sigma)(t-s)} d s,\right. \\
& \left.\quad \delta\left\|\varphi^{j-1}\left(x_{1}\right)-\varphi^{j-1}\left(x_{2}\right)\right\|_{\sigma} \int_{t}^{\infty} e^{(\beta-\sigma)(t-s)} d s\right\} \\
& =\max \left\{e^{(\alpha-\sigma) t}\left\|x_{1}-x_{2}\right\|+\frac{\delta}{\sigma-\alpha}\left\|\varphi^{j-1}\left(x_{1}\right)-\varphi^{j-1}\left(x_{2}\right)\right\|_{\sigma}, \frac{\delta}{\beta-\sigma}\left\|\varphi^{j-1}\left(x_{1}\right)-\varphi^{j-1}\left(x_{2}\right)\right\|_{\sigma}\right\} \\
& \leq\left\|x_{1}-x_{2}\right\|+\kappa\left\|\varphi^{j-1}\left(x_{1}\right)-\varphi^{j-1}\left(x_{2}\right)\right\|_{\sigma} .
\end{aligned}
$$

Now, since the right hand side of the inequality does not depend on $t$, take the supremum over $t$ and obtain

$$
\begin{aligned}
\left\|\varphi^{j}\left(x_{1}\right)-\varphi^{j}\left(x_{2}\right)\right\|_{\sigma} & \leq\left\|x_{1}-x_{2}\right\|+\kappa\left\|\varphi^{j-1}\left(x_{1}\right)-\varphi^{j-1}\left(x_{2}\right)\right\|_{\sigma} \\
& \leq\left\|x_{1}-x_{2}\right\|+\kappa\left\|x_{1}-x_{2}\right\|+\kappa^{2}\left\|\varphi^{j-2}\left(x_{1}\right)-\varphi^{j-2}\left(x_{2}\right)\right\|_{\sigma} \\
& \leq\left\|x_{1}-x_{2}\right\|+\kappa\left\|x_{1}-x_{2}\right\|+\ldots+\kappa^{j}\left\|\varphi^{0}\left(x_{1}\right)-\varphi^{0}\left(x_{2}\right)\right\|_{\sigma} \\
& =\left(1+\kappa+\kappa^{2}+\ldots+\kappa^{j}\right)\left\|x_{1}-x_{2}\right\| \leq \frac{1}{1-\kappa}\left\|x_{1}-x_{2}\right\| .
\end{aligned}
$$

Since $\Phi_{z_{0}}(x)=y_{0}+Q \varphi(0)$, we can obtain an estimate for $\Phi^{j}$. For the exact manifold, from [5] we have $\operatorname{Lip}\left(\Phi_{z_{0}}\right) \leq \frac{\delta}{\beta-\alpha-\delta}$.

Lemma 4.2. Let $\Phi^{j}$ be defined as in (4.2) and $x_{1}, x_{2} \in X$.

$$
\left\|\Phi^{j}\left(x_{1}\right)-\Phi^{j}\left(x_{2}\right)\right\| \leq \frac{\delta}{\beta-\sigma} \frac{1}{1-\kappa}\left\|x_{1}-x_{2}\right\| .
$$


Proof. Apply Lemma 4.1 to find that

$$
\begin{aligned}
\left\|\Phi^{j}\left(x_{1}\right)-\Phi^{j}\left(x_{2}\right)\right\| & =\left\|\int_{0}^{\infty} e^{-s B}\left[G\left(\varphi^{j-1}\left(x_{1}\right)(s)+z\left(s, z_{0}\right)\right)-G\left(\varphi^{j-1}\left(x_{2}\right)(s)+z\left(s, z_{0}\right)\right)\right] d s\right\| \\
& \leq \int_{0}^{\infty} e^{-s \beta} \delta\left\|\varphi^{j-1}\left(x_{1}\right)(s)-\varphi^{j-1}\left(x_{2}\right)(s)\right\| d s \\
& =\int_{0}^{\infty} e^{-s \beta} e^{s \sigma} \delta e^{-s \sigma}\left\|\varphi^{j-1}\left(x_{1}\right)(s)-\varphi^{j-1}\left(x_{2}\right)(s)\right\| d s \\
& \leq \delta\left\|\varphi^{j-1}\left(x_{1}\right)-\varphi^{j-1}\left(x_{2}\right)\right\|_{\sigma} \int_{0}^{\infty} e^{(\sigma-\beta) s} d s \\
& =\frac{\delta}{\beta-\sigma}\left\|\varphi^{j-1}\left(x_{1}\right)-\varphi^{j-1}\left(x_{2}\right)\right\|_{\sigma} \leq \frac{\delta}{\beta-\sigma} \frac{1}{1-\kappa}\left\|x_{1}-x_{2}\right\| .
\end{aligned}
$$

We have an analogous result for $\Psi$, the proof of which is similar.

Lemma 4.3. Let $\Psi^{j}$ be defined as in (4.2) and $y_{1}, y_{2} \in Y$.

$$
\left\|\Psi^{j}\left(y_{1}\right)-\Psi^{j}\left(y_{2}\right)\right\| \leq \frac{\delta}{\sigma-\alpha} \frac{1}{1-\kappa}\left\|y_{1}-y_{2}\right\| .
$$

Since we now have both estimates for $\Phi^{j}$ and $\Psi^{j}$, we are ready to show that $\Sigma^{j_{1}, j_{2}}$ is a contraction mapping.

\section{Proposition 4.4. If}

$$
4 \delta<\beta-\alpha,
$$

then $\Sigma^{j_{1}, j_{2}}$ defined in (4.3) is a contraction mapping with the rate $\frac{\kappa}{1-\kappa}$, where $\kappa=\max \left\{\frac{\delta}{\beta-\sigma}, \frac{\delta}{\sigma-\alpha}\right\}$ for any $\sigma \in(\alpha+2 \delta, \beta-2 \delta)$.

Proof. By Lemma 4.2 and 4.3 .

$$
\begin{aligned}
\left\|\Sigma^{j_{1}, j_{2}}\left(x_{1}, y_{1}\right)-\Sigma^{j_{1}, j_{2}}\left(x_{2}, y_{2}\right)\right\| & =\max \left\{\left\|\Phi^{j}\left(x_{1}\right)-\Phi^{j}\left(x_{2}\right)\right\|,\left\|\Psi^{j}\left(y_{1}\right)-\Psi^{j}\left(y_{2}\right)\right\|\right\} \\
& \leq \frac{\kappa}{1-\kappa} \max \left\{\left\|x_{1}-x_{2}\right\|,\left\|y_{1}-y_{2}\right\|\right\} .
\end{aligned}
$$

By (4.5) and the assumption that $\sigma \in(\alpha+2 \delta, \beta-2 \delta)$, we have $\kappa<1 / 2$, and hence

$$
\frac{\kappa}{1-\kappa}<1
$$

Therefore $\Sigma^{j_{1}, j_{2}}$ is a contraction mapping.

Proposition 4.5. $\Sigma^{j_{1}, j_{2}}$ has as its fixed point the intersection of the graphs of $\Psi^{j_{1}}$ and $\Phi^{j_{2}}$.

The proof is as for $\Sigma$ in $[5$.

4.3. Error estimate. Let $z_{0}^{*}$ be the fixed point of $\Sigma^{j_{1}, j_{2}}$, and $z_{0}^{+}$the exact tracking solution (fixed point of $\Sigma$ ). We seek an estimate for $\left\|z_{0}^{+}-z_{0}^{*}\right\|$. We start with two lemmas.

Lemma 4.6. Let $z_{0} \in Z$. For any $x \in X$ and positive integer $j$, we have

$$
\left\|\varphi(x)-\varphi^{j}(x)\right\|_{\sigma} \leq \frac{\kappa^{j}}{1-\kappa}\left\|\varphi^{1}(x)-\varphi^{0}(x)\right\|_{\sigma},
$$

where $\varphi$ is the fixed point of $\mathcal{T}_{z_{0}}(\varphi, x)$ and $\kappa=\max \left\{\frac{\delta}{\beta-\sigma}, \frac{\delta}{\sigma-\alpha}\right\}$. 
Proof. Let $m$ be an integer such that $m>j$. From [5], we know that $\operatorname{Lip}(\mathcal{T}) \leq \kappa$ and by the gap condition, $\kappa<1$,

$$
\begin{aligned}
\left\|\varphi^{m}(x)-\varphi^{j}(x)\right\|_{\sigma} & \leq \Sigma_{k=j+1}^{m}\left\|\varphi^{k}(x)-\varphi^{k-1}(x)\right\|_{\sigma} \\
& \leq \Sigma_{k=j+1}^{m} \kappa^{k-1}\left\|\varphi^{1}(x)-\varphi^{0}(x)\right\|_{\sigma} \leq \frac{\kappa^{j}}{1-\kappa}\left\|\varphi^{1}(x)-\varphi^{0}(x)\right\|_{\sigma} .
\end{aligned}
$$

Since the last inequality is independent of $m$, take $m \rightarrow \infty$ to obtain

$$
\left\|\varphi(x)-\varphi^{j}(x)\right\|_{\sigma} \leq \frac{\kappa^{j}}{1-\kappa}\left\|\varphi^{1}(x)-\varphi^{0}(x)\right\|_{\sigma} .
$$

Since $\Phi_{z_{0}}(x):=y_{0}+Q \varphi(x)(0)$, the estimate for $\left\|\Phi(x)-\Phi^{j}(x)\right\|$ is straightforward.

Lemma 4.7. Under the same assumption above, one has

$$
\left\|\Phi(x)-\Phi^{j}(x)\right\| \leq \frac{\kappa^{j}}{1-\kappa}\left\|\varphi^{1}(x)-\varphi^{0}(x)\right\|_{\sigma} .
$$

Proof. By the definition of $\Phi$ and the previous lemma,

$$
\begin{aligned}
\left\|\Phi(x)-\Phi^{j}(x)\right\| & \leq\left\|Q \varphi(x)(0)-Q \varphi^{j}(x)(0)\right\| \\
& \leq\left\|\varphi(x)-\varphi^{j}(x)\right\|_{\sigma} \leq \frac{\kappa^{j}}{1-\kappa}\left\|\varphi^{1}(x)-\varphi^{0}(x)\right\|_{\sigma} .
\end{aligned}
$$

We have an analogous result for $\Psi$, the proof of which is similar.

Lemma 4.8. Under the same assumption above, one has

$$
\left\|\Psi(y)-\Psi^{j}(y)\right\| \leq \frac{\kappa^{j}}{1-\kappa}\left\|\psi^{1}(y)-\psi^{0}(y)\right\|_{\sigma} .
$$

By Lemmas 4.7 and 4.8, one can deduce that

$$
\left\|\Sigma(z)-\Sigma^{j_{1}, j_{2}}(z)\right\| \leq \frac{\kappa^{j}}{1-\kappa} \max \left\{\kappa^{j_{2}-j}\left\|\varphi^{1}(x)-\varphi^{0}(x)\right\|_{\sigma}, \kappa^{j_{1}-j}\left\|\psi^{1}(y)-\psi^{0}(y)\right\|_{\sigma}\right\},
$$

where $j=\min \left\{j_{1}, j_{2}\right\}$. We are ready to give an estimate for $\left\|z_{0}^{+}-z_{0}^{*}\right\|$.

Proposition 4.9. Let $z_{0}^{+}$be the fixed point of $\Sigma$ and $z_{0}^{*}$ be the fixed point of $\Sigma^{j_{1}, j_{2}}$. Then

$$
\left\|z_{0}^{+}-z_{0}^{*}\right\| \leq c_{j_{1}, j_{2}} \frac{\kappa^{j}}{1-2 \kappa}
$$

where $j=\min \left\{j_{1}, j_{2}\right\}$ and $c_{j_{1}, j_{2}}=\max \left\{\kappa^{j_{2}-j}\left\|\varphi^{1}(x)-\varphi^{0}(x)\right\|_{\sigma}, \kappa^{j_{1}-j} \| \psi^{1}(y)-\right.$ $\left.\psi^{0}(y) \|_{\sigma}\right\}$.

Proof. By (4.6) and Proposition 4.4,

$$
\begin{aligned}
\left\|z_{0}^{+}-z_{0}^{*}\right\| & =\left\|\Sigma\left(z_{0}^{+}\right)-\Sigma^{j_{1}, j_{2}}\left(z_{0}^{*}\right)\right\| \leq\left\|\Sigma\left(z_{0}^{+}\right)-\Sigma^{j_{1}, j_{2}}\left(z_{0}^{+}\right)\right\|+\left\|\Sigma^{j_{1}, j_{2}}\left(z_{0}^{+}\right)-\Sigma^{j_{1}, j_{2}}\left(z_{0}^{*}\right)\right\| \\
& \leq c_{j_{1}, j_{2}} \frac{\kappa^{j}}{1-\kappa}+\frac{\kappa}{1-\kappa}\left\|z_{0}^{+}-z_{0}^{*}\right\| .
\end{aligned}
$$

Therefore, we have

$$
\left\|z_{0}^{+}-z_{0}^{*}\right\| \leq c_{j_{1}, j_{2}} \frac{\kappa^{j}}{1-2 \kappa} .
$$

For simplicity, we denote $\Sigma^{j}:=\Sigma^{j, j}$ in the following numerical tests. 


\begin{tabular}{|l|l|}
\hline$z^{i+1}$ & Error \\
\hline$z^{0}$ & 3.5429 \\
\hline$z^{1}$ & $2.4 \mathrm{E}-2$ \\
\hline$z^{2}$ & $8.4 \mathrm{E}-5$ \\
\hline$z^{3}$ & $2.9 \mathrm{E}-7$ \\
\hline$z^{4}$ & $1.0 \mathrm{E}-9$ \\
\hline$z^{5}$ & $3.3 \mathrm{E}-11$ \\
\hline
\end{tabular}

(A)

\begin{tabular}{|l|l|}
\hline$\Sigma^{j}$ & Error \\
\hline$\Sigma^{1}$ & $4.97 \mathrm{E}-4$ \\
\hline$\Sigma^{2}$ & $8.49 \mathrm{E}-6$ \\
\hline$\Sigma^{3}$ & $1.85 \mathrm{E}-7$ \\
\hline$\Sigma^{4}$ & $4.50 \mathrm{E}-9$ \\
\hline$\Sigma^{5}$ & $1.16 \mathrm{E}-10$ \\
\hline$\Sigma^{6}$ & $3.25 \mathrm{E}-11$ \\
\hline
\end{tabular}

(B)

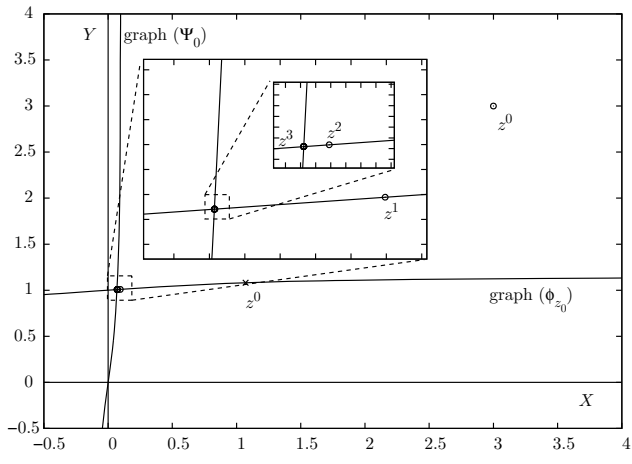

(c)

Figure 4. (A) This table shows the convergence of the tracking solution for (1.13) using SIMP for fixed $j=15$ and $z^{i+1}:=\Sigma^{j}\left(z^{i}\right)$ with $h=0.01$. (B) This table shows the relation between $j$ and the absolute error. (C) Convergence for data in (A) in phase space.

\subsection{Application.}

4.4.1. Test problem. In this section, we demonstrate the computation of the tracking solution for the test problem (1.13). In this test, $\left(x_{0}, y_{0}\right)=(1,1)$ and $p=10$ and hence by (1.16), the exact tracking solution is

$$
\left(\begin{array}{l}
u_{0}^{+} \\
v_{0}^{+}
\end{array}\right)=\left(\begin{array}{c}
\frac{1}{10 \sqrt{2}} \\
1+\frac{1}{10} \tan ^{-1}\left(\frac{1}{10 \sqrt{2}}\right)
\end{array}\right) \approx\left(\begin{array}{c}
7.0711 e-02 \\
1.0070
\end{array}\right) .
$$

By Proposition 4.4, for fixed $j$, the Lipschitz constant for $\Sigma^{j}$ is

$$
\frac{\kappa}{1-\kappa} \text {. }
$$

In the test problem, $\alpha=-1, \beta=1$, and $\delta<1 / p$ and hence $\kappa<1 / p$. In this case, $p=10$, and therefore $\operatorname{Lip}\left(\Sigma^{j}\right)<1 / 9$. As Table 4(A) shows, in the first two iterations we observe that the error decreases roughly by a factor of 0.01 which confirms $\operatorname{Lip}\left(\Sigma^{j}\right)<1 / 9$.

To verify Proposition 4.9. we will vary $j$ and observe how the error depends on $j$. As in Table 4(B), we can see that the error decreases roughly by a factor of 0.1 as $j$ increases which also confirms Proposition 4.9 that the rate of the convergence is approximately $\kappa(\approx 1 / 10)$.

\section{Comparison Between the tracking solution And the Projected one}

Given an initial condition $z_{0}$, it is natural to take $\tilde{z_{0}}:=\left(0, y_{0}\right)$, the linear projection onto the unstable eigenspace, as the approximate tracking solution since $Y$ is tangent to the inertial manifold. However, the projected initial condition may have the wrong long time behavior as Figure 5(A) shows. Moreover, even if it captures the correct long time behaviors, the tracking rate is not optimal compared to the tracking rate for the tracking solution.

By Proposition 1.2, one has that

$$
\left\|z\left(t, z_{0}\right)-z\left(t, z_{0}^{+}\right)\right\| \leq e^{(\alpha+\delta) t}\left\|x_{0}-x_{0}^{+}\right\|, \forall t \geq 0 .
$$




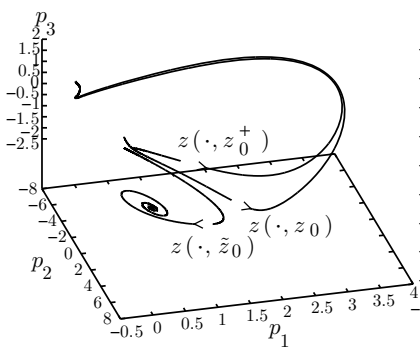

(A)

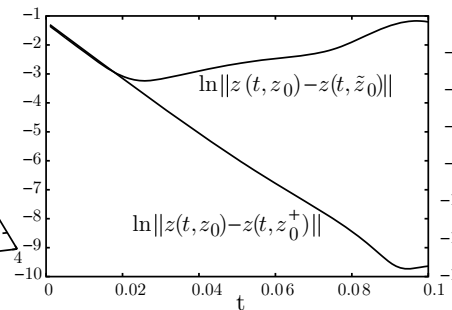

(B)

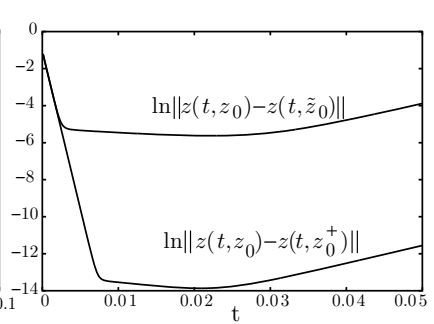

(C)

Figure 5. (A) For (2.17), we have that $z_{0}=(0.12,0.5,0.5)$ and $\tilde{z_{0}}=(0.12,0.5,0.0)$ and the tracking solution $z_{0}^{+}=$ $(0.2603,0.5225,-0.00364)$, found as the fixed point of $\Sigma^{6}$. (B) For (2.17). (C) For 8-mode KSE.

Thus, the tracking rate for $z_{0}^{+}$is $\alpha+\delta$. To estimate for the tracking rate for $\tilde{z_{0}}$, we need the so-called cone invariance property in [5]. It is a stronger version of the squeezing property (where the gap condition does not hold), which was originally introduced for the Navier-Stokes equation in [13] and improved in [9].

Proposition 5.1 (Cone Invariance Property). Consider the system (1.1) and assume (1.2)-(1.4). If $z_{1} \neq z_{2}$ and we denote $u(t)=x\left(t, z_{1}\right)-x\left(t, z_{2}\right)$ and $v(t)=$ $y\left(t, z_{1}\right)-y\left(t, z_{2}\right)$, then one and only one of the following holds:

(i) $\|v(t)\|<\|u(t)\|, \forall t \in \mathbb{R}$;

(ii) $\|v(t)\|>\|u(t)\|, \forall t \in \mathbb{R}$;

(iii) There exists $t_{0} \in \mathbb{R}$ such that

$$
\left\{\begin{array}{l}
\|v(t)\|<\|u(t)\|, \forall t<t_{0}, \\
\|v(t)\|=\|u(t)\|, \forall t=t_{0}, \\
\|v(t)\|>\|u(t)\|, \forall t>t_{0} .
\end{array}\right.
$$

In addition to the Cone Invariance Property, we need for the next result an exponential dichotomy in reverse time. Since $A$ and $B$ are bounded, we have

$$
\left\|e^{t A}\right\| \leq K e^{a t}, \quad\left\|e^{-t B}\right\| \leq K e^{-b t}, \forall t \leq 0
$$

for some $K \geq 1$, and some $a \leq \alpha$ and $b \geq \beta$. For instance, we may take $a=\|A\|$, $b=\|B\|$, and $K=1$, though this may not be the optimal choice.

Lemma 5.2. For any $z_{0} \in Z$, either

$$
\left\|z\left(t, z_{0}\right)-z\left(t, \tilde{z_{0}}\right)\right\| \leq e^{(\alpha+\delta) t}\left\|x_{0}\right\|, \forall t \geq 0,
$$

or there exists a $t_{0} \in(0, \infty)$ such that

$$
\left\|z\left(t, z_{0}\right)-z\left(t, \tilde{z_{0}}\right)\right\| \leq\left\{\begin{array}{l}
e^{(\alpha+\delta) t}\left\|x_{0}\right\|, \forall t \in\left[0, t_{0}\right] \\
K e^{(b+K \delta)\left(t-t_{0}\right)}\left\|x_{0}\right\|, \forall t \in\left(t_{0}, \infty\right) .
\end{array}\right.
$$

Proof. Denote $u(t)=x\left(t, z_{0}\right)-x\left(t, \tilde{z_{0}}\right)$ and $v(t)=y\left(t, z_{0}\right)-y\left(t, \tilde{z_{0}}\right)$. It is clear that $0=\|v(0) \mid \leq\| u(0)\|=\| x_{0} \|$ and thus by Proposition [5.1, either

(i) $\|v(t)\| \leq\|u(t)\|, \quad \forall t \in \mathbb{R}$ 
or (ii) there exists $t_{0} \in \mathbb{R}$ such that (5.2) holds. By variation of constants, one has $x\left(t, z_{0}\right)=e^{t A} x_{0}+\int_{0}^{t} e^{(t-s) A} F\left(z\left(s, z_{0}\right)\right) d s$, and $x\left(t, \tilde{z_{0}}\right)=\int_{0}^{t} e^{(t-s) A} F\left(z\left(s, \tilde{z_{0}}\right)\right) d s$.

If (i) holds, then

$$
\left\|z\left(t, z_{0}\right)-z\left(t, \tilde{z_{0}}\right)\right\|=\|u(t)\| \leq e^{\alpha t}\left\|x_{0}\right\|+\delta \int_{0}^{t} e^{(t-s) A}\|u(s)\| d s .
$$

By the Gronwall inequality, one has that

$$
\|u(t)\| \leq e^{(\alpha+\delta) t}\left\|x_{0}\right\|, \forall t \geq 0 .
$$

This proves the first part of the lemma.

Now if (ii) holds, for $t \leq t_{0}$, by the above estimate, we have

$$
\left\|z\left(t, z_{0}\right)-z\left(t, \tilde{z_{0}}\right)\right\| \leq e^{(\alpha+\delta) t}\left\|x_{0}\right\| .
$$

By variation of constants, one has

$$
v(t)=e^{\left(t-t_{0}\right) B} v\left(t_{0}\right)+\int_{t_{0}}^{t} e^{(t-s) B}\left[H\left(z\left(s, z_{0}\right)\right)-H\left(z\left(s, \tilde{z_{0}}\right)\right)\right] d s,
$$

which is bounded by

$$
\|v(t)\| \leq K e^{\left(t-t_{0}\right) b}\left\|v\left(t_{0}\right)\right\|+K \delta \int_{t_{0}}^{t} e^{(t-s) b}\|v(s)\| d s .
$$

Because of (1.4), it is easy to verify that $\alpha+\delta-\beta-K \delta<0$. Hence, by the Gronwall inequality and the fact $\left\|v\left(t_{0}\right)\right\|=\left\|u\left(t_{0}\right)\right\|$ we have for $t>t_{0}$,

$$
\begin{aligned}
\|v(t)\| & \leq K\left\|u\left(t_{0}\right)\right\| e^{(b+K \delta)\left(t-t_{0}\right)} \leq K e^{(b+K \delta)\left(t-t_{0}\right)} e^{(\alpha+\delta) t_{0}}\left\|x_{0}\right\| \\
& =K e^{(b+K \delta) t+(\alpha+\delta-\beta-K \delta) t_{0}}\left\|x_{0}\right\| \leq K e^{(b+K \delta) t}\left\|x_{0}\right\| .
\end{aligned}
$$

This lemma suggests that the tracking rate for $\tilde{z_{0}}$ is the same as the one for $z_{0}^{+}$ over a short period of time, namely $\left[0, t_{0}\right]$ for some $t_{0}$, but after that, the tracking rate becomes worse than $z_{0}^{+}$.

To show this result numerically, we consider both (2.17) with $\gamma=25, \operatorname{dim}(X)=1$ and $\operatorname{dim}(Y)=2$ with the initial condition $z_{0}$ chosen at random within a ball of radius 0.4 and the 8-mode Galerkin approximation of the KSE with $\gamma=32$, $\operatorname{dim}(X)=4$, and $\operatorname{dim}(Y)=4$ and the initial condition $z_{0}$ chosen at random from a ball within radius 0.5 . Figure 5(B) and (C) compare the rates of attractions for the projected and tracking initial data. Moreover, we compute their tracking rates via the simple linear regressions for these data sets in a short period of time (0.1 and .008 for (2.17) and 8-mode Galerkin approximation of the KSE) and they are -88.40 and 12.59 for (2.17) and -1640.45 and -400.04 for 8-mode Galerkin approximation of the KSE. The gap in eigenvalues is the interval $(-99,21)$ for (2.17) and $(-1700,-512)$ for 8 -mode Galerkin approximation of the KSE. To ensure that this is not a special case, we repeat the above process with 10000 randomly chosen initial conditions and plot the tracking rates as shown in Figure 6. Observe that the data for tracking solutions are mainly close to the lower bound of the gap, while the data for projected initial conditions are mainly close to the upper bound of the gap. 


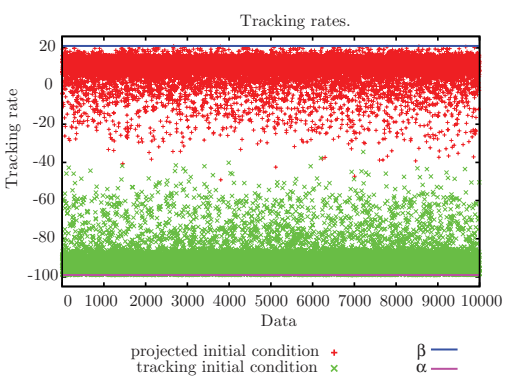

(A) 2.17)

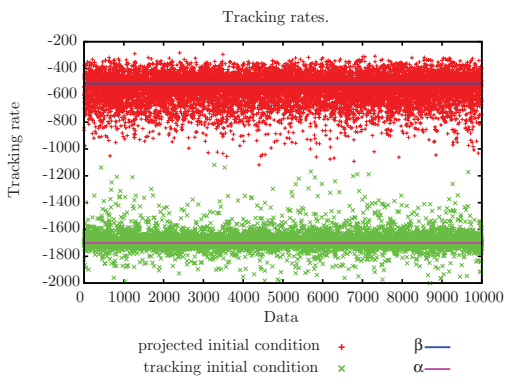

(B) 8-mode KSE

FIGURE 6. Tracking rate for tracking solution $(x)$ is near the lower bound of the gap while the other $(+)$ is near the upper bound of the gap.

\section{REFERENCES}

[1] Peter W. Bates and Kening Lu, A Hartman-Grobman theorem for the Cahn-Hilliard and phase-field equations, J. Dynam. Differential Equations 6 (1994), no. 1, 101-145, DOI 10.1007/BF02219190. MR.1262725 (94m:35280)

[2] Peter W. Bates, Kening Lu, and Chongchun Zeng, Existence and persistence of invariant manifolds for semiflows in Banach space, Mem. Amer. Math. Soc. 135 (1998), no. 645, viii+129, DOI 10.1090/memo/0645. MR1445489 (99b:58210)

[3] Peter W. Bates, Kening Lu, and Chongchun Zeng, Invariant foliations near normally hyperbolic invariant manifolds for semiflows, Trans. Amer. Math. Soc. 352 (2000), no. 10, 4641-4676, DOI 10.1090/S0002-9947-00-02503-4. MR 1675237(2001b:37031)

[4] Xavier Cabré, Ernest Fontich, and Rafael de la Llave, The parameterization method for invariant manifolds. III. Overview and applications, J. Differential Equations 218 (2005), no. 2, 444-515, DOI 10.1016/j.jde.2004.12.003. MR2177465(2007b:37057)

[5] Nelson Castañeda and Ricardo Rosa, Optimal estimates for the uncoupling of differential equations, J. Dynam. Differential Equations 8 (1996), no. 1, 103-139, DOI 10.1007/BF02218616. MR1388166 (97d:34032)

[6] Shui-Nee Chow, Xiao-Biao Lin, and Kening Lu, Smooth invariant foliations in infinitedimensional spaces, J. Differential Equations 94 (1991), no. 2, 266-291, DOI 10.1016/00220396(91)90093-O. MR1137616 (92k:58210)

[7] Y.-M. Chung. FOLI8PAK software package. The Dynamical Systems Web Software List (http://www.dynamicalsystems.org/sw/).

[8] Stephen M. Cox and A. J. Roberts, Initial conditions for models of dynamical systems, Phys. D 85 (1995), no. 1-2, 126-141, DOI 10.1016/0167-2789(94)00201-Z. MR.1339235(96e:34072)

[9] C. Foias, O. Manley, and R. Temam, Modelling of the interaction of small and large eddies in two-dimensional turbulent flows (English, with French summary), RAIRO Modél. Math. Anal. Numér. 22 (1988), no. 1, 93-118. MR934703 (89h:76022)

[10] C. Foias, B. Nicolaenko, G. R. Sell, and R. Temam, Inertial manifolds for the KuramotoSivashinsky equation and an estimate of their lowest dimension, J. Math. Pures Appl. (9) 67 (1988), no. 3, 197-226. MR964170 (90e:35137)

[11] Ciprian Foias, George R. Sell, and Roger Temam, Inertial manifolds for nonlinear evolutionary equations, J. Differential Equations 73 (1988), no. 2, 309-353, DOI 10.1016/00220396(88)90110-6. MR943945 (89e:58020)

[12] Ciprian Foias, George R. Sell, and Edriss S. Titi, Exponential tracking and approximation of inertial manifolds for dissipative nonlinear equations, J. Dynam. Differential Equations 1 (1989), no. 2, 199-244, DOI 10.1007/BF01047831. MR1010966 (90k:35031)

[13] C. Foiaş and R. Temam, Some analytic and geometric properties of the solutions of the evolution Navier-Stokes equations, J. Math. Pures Appl. (9) 58 (1979), no. 3, 339-368. MR544257 (81k:35130) 
[14] M. S. Jolly, I. G. Kevrekidis, and E. S. Titi, Approximate inertial manifolds for the KuramotoSivashinsky equation: analysis and computations, Phys. D 44 (1990), no. 1-2, 38-60, DOI 10.1016/0167-2789(90)90046-R. MR.1069671 (91f:35217)

[15] M. S. Jolly and R. Rosa, Computation of non-smooth local centre manifolds, IMA J. Numer. Anal. 25 (2005), no. 4, 698-725, DOI 10.1093/imanum/dri013. MR2170520 (2006g:37135)

[16] M. S. Jolly, R. Rosa, and R. Temam, Accurate computations on inertial manifolds, SIAM J. Sci. Comput. 22 (2000), no. 6, 2216-2238 (electronic), DOI 10.1137/S1064827599351738. MR $1856310(2002 \mathrm{k}: 35044)$

[17] M. S. Jolly, R. Rosa, and R. Temam, Evaluating the dimension of an inertial manifold for the Kuramoto-Sivashinsky equation, Adv. Differential Equations 5 (2000), no. 1-3, 31-66. MR.1734536(2001a:37127)

[18] U. Kirchgraber and K. J. Palmer, Geometry in the Neighborhood of Invariant Manifolds of Maps and Flows and Linearization, Pitman Research Notes in Mathematics Series, vol. 233, Longman Scientific \& Technical, Harlow, 1990. MR1068954 (91k:58115)

[19] B. Krauskopf, H. M. Osinga, E. J. Doedel, M. E. Henderson, J. Guckenheimer, A. Vladimirsky, M. Dellnitz, and O. Junge, A survey of methods for computing (un) stable manifolds of vector fields, Internat. J. Bifur. Chaos Appl. Sci. Engrg. 15 (2005), no. 3, 763-791, DOI 10.1142/S0218127405012533. MR2136745(2006h:37116)

[20] Kening Lu, A Hartman-Grobman theorem for scalar reaction-diffusion equations, J. Differential Equations 93 (1991), no. 2, 364-394, DOI 10.1016/0022-0396(91)90017-4. MR1125224 (92k:35147)

[21] Yves Nievergelt, Aitken's and Steffensen's accelerations in several variables, Numer. Math. 59 (1991), no. 3, 295-310, DOI 10.1007/BF01385782. MR.1106386 (92a:65172)

[22] Christian Pötzsche and Martin Rasmussen, Computation of nonautonomous invariant and inertial manifolds, Numer. Math. 112 (2009), no. 3, 449-483, DOI 10.1007/s00211-009-02159. MR2501313 (2010a:65135)

[23] A. J. Roberts, Appropriate initial conditions for asymptotic descriptions of the long term evolution of dynamical systems, J. Austral. Math. Soc. Ser. B 31 (1989), no. 1, 48-75, DOI 10.1017/S0334270000006470. MR1002091 (90h:58075)

[24] A. J. Roberts, Computer algebra derives correct initial conditions for low-dimensional dynamical models, Computer Physics Communication 126 (2000), 187-206.

[25] James C. Robinson, Computing inertial manifolds, Discrete Contin. Dyn. Syst. 8 (2002), no. 4, 815-833, DOI 10.3934/dcds.2002.8.815. MR1920645(2003g:37142)

[26] Ricardo Rosa, Approximate inertial manifolds of exponential order, Discrete Contin. Dynam. Systems 1 (1995), no. 3, 421-448, DOI 10.3934/dcds.1995.1.421. MR1355883 (96j:34114)

[27] Roger Temam and Xiao Ming Wang, Estimates on the lowest dimension of inertial manifolds for the Kuramoto-Sivashinsky equation in the general case, Differential Integral Equations 7 (1994), no. 3-4, 1095-1108. MR:1270121 (95b:35219)

Department of Mathematics, University of Kansas, Lawrence, Kansas 66045

E-mail address: ychung@wm.edu

Department of Mathematics, Indiana University, Bloomington, Indiana 47405

E-mail address: msjolly@indiana.edu 